\title{
Marc Cluzel
}

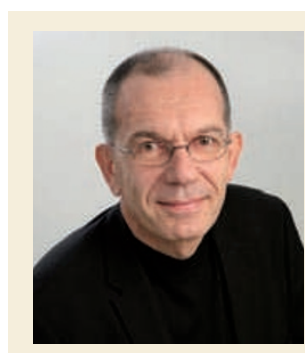

Executive Vice President, R\&D, Sanofi-Aventis, Paris, France. In November 2009, Marc Cluzel was appointed Executive Vice President (VP) of R\&D at Sanofi-Aventis. He has a medical and biochemical education from the University of Montpellier, France, and was a Visiting Assistant Professor at the Johns Hopkins University, Baltimore, USA, then a Research Associate at Guy's Hospital in London, UK, before joining Sanofi-Aventis in 1991. He was appointed VP of International Development in 2001, Senior VP of International Development in 2005 and Senior VP of RED in January 2007.

What have been the major changes in the company's strategy since Chris Viehbacher became Chief Executive Officer in December 2008 , in particular in $R \& D$ ?

To address the challenge that the classical blockbuster model can no longer support large pharmaceutical companies, Chris Viehbacher has introduced a more global vision for Sanofi-Aventis to become a global, diversified health-care company focused on patients' needs.

In terms of $\mathrm{R} \& \mathrm{D}$, from the beginning Chris has been clear that innovation is the main driver and needs to be at the heart of the organization as an inherent part of our culture. However - and this is a new viewpoint for the company - just because a project is innovative does not mean that it will be funded. An innovative project has to go through the same selection procedure for investment as any product in the company.

\section{What do you think are the most important factors for developing an innovative R\&D culture?}

One of the most important factors is the need to collaborate with the outside world. The traditional pharmaceutical model was attracting innovation from the outside but, once the innovation was brought in, it was digested by the company. We need to develop an innovative culture that can allow us to quickly identify disruptive science (an example of which is the 1969 invention of the charge-coupled device which revolutionized photography in the late 1990s, won the 2009 Nobel Prize in Physics and helped achieve a milestone in diagnostics) and apply it to benefit the pharmaceutical industry.

Another factor is that we need to align the culture of the company with the patient-centred approach. When we now discuss a new approach to treatment, we are not considering patients as a group, but a patient as an individual. However, if you start to put your R\&D resources to meet the needs of an individual, you very quickly see that you cannot do it all by yourself. Providing health care for an individual involves more than just developing a drug. For example, you need to identify the right patient for a treatment and you need diagnostic tools and monitoring devices to know if it is working. The pharmaceutical industry cannot do this by itself, so we need to work in networks. This means partnering with outside groups. In this case, partnering is not taking ideas or products from outside and internalizing them; it is sharing ideas and activities, collaborating in R\&D and, at the end, sharing profits. In addition, you have to add flexibility because no one can predict what pharmacy will be like in 2020 . We need to have flexibility in the culture of the organization to quickly identify promising $R \& D$ routes.

\section{You cannot build a} relationship in the long term just by funding; you have to share ideas, resources and strategy.
How is Sanofi-Aventis engaging with external partners for the discovery and development of novel therapies?
We have announced a lot of partnering in the past year. For example, in discovery research in March 2009 we signed a strategic alliance agreement with the Salk Institute for Biological Studies in San Diego, California. With Salk we are partners in basic science and we have set up an institute-wide

system of grants, primarily for regenerative medicine, that Salk scientists can apply for. The recipients of the grant are chosen by a mixed jury and the aim is that Sanofi-Aventis scientists can learn from Salk scientists and vice versa. In terms of partnership, the alliance with Salk highlights that if you are part of a network you have to clearly define the added value that you bring. You cannot build a relationship in the long term just by funding; you have to share ideas, resources and strategy. It is important to interact and work together, rather than taking an idea from outside and developing it yourself. I think that is one of the big changes we have made in terms of partnering with outside groups.

We will continue to license products in a traditional way. For example, we announced a licensing agreement on 3 December 2009 with Rockefeller University for a novel monoclonal antibody that targets certain specific forms of the amyloid- $\beta$ parenchymal plaque for the treatment of Alzheimer's disease. Another licensing deal with Exelixis, announced in May 2009, combines the licensing of two anticancer drug candidates with discovery research collaboration.

\section{Some pharmaceutical companies have recently announced their intention to move} out of cardiovascular disease research. Are there any therapeutic areas that Sanofi-Aventis is aiming to focus on more (or less) in the future?

I think that linking the future of $\mathrm{R} \& \mathrm{D}$ to therapeutic areas is the wrong approach. Considering the needs of an individual involves a holistic approach to disease. For example, myocardial infarction occurs more often in people over the age of 50 years, so our research in this area aims to address both cardiovascular disease and the ageing component. Also, to date, diseases have been classified by symptoms. This is problematic because you may think you are looking at one disease when in fact you are observing another. Now we are moving towards better characterization of disease - for example, by defining the underlying molecular mechanism — and so thinking about disease in terms of a therapeutic area is no longer sufficient. 